\title{
Awake and fully mobile patients on cardiac extracorporeal life support
}

\author{
Darryl Abrams ${ }^{1}$, A. Reshad Garan ${ }^{2}$, Daniel Brodie ${ }^{1}$ \\ ${ }^{1}$ Division of Pulmonary, Allergy, and Critical Care, ${ }^{2}$ Division of Cardiology, NewYork-Presbyterian Hospital/Columbia University Medical Center, \\ New York, USA \\ Correspondence to: Darryl Abrams, MD. 622 W.168 ${ }^{\text {th }}$ St, PH 8E 101, New York, NY 10032, USA. Email: da2256@cumc.columbia.edu.
}

Early mobilization of critically ill patients is increasingly being recognized as not only safe and feasible, but also as a potential means of optimizing outcomes in the intensive care unit (ICU). With the rapidly expanding use of extracorporeal life support (ECLS) for severe cardiopulmonary failure, there is a growing interest in the application of early mobilization to this patient population, which has been shown to be safe and feasible in select patient populations. However, some patients receiving ECLS support may benefit more than others. For instance, early mobilization may be particularly beneficial in patients awaiting heart or lung transplantation, as maintenance of physical conditioning may be an important component of a patient's transplant candidacy. The ability to engage critically ill patients in active physical therapy and early mobilization necessarily involves minimization of sedation and is often further facilitated by a strategy that favors endotracheal extubation. Whether an awake, extubated and mobile strategy can be applied in any given patient is often dictated by the severity of the underlying disease and the amount of extracorporeal support required. Additionally, whether this approach is superior to usual care, which patients might benefit or be harmed, and which patient characteristics are most likely to predict success of this strategy, are areas of ongoing investigation.

Keywords: Early mobilization; extracorporeal membrane oxygenation (ECMO); physical therapy; ambulation; transplantation

Submitted May 17, 2018. Accepted for publication Jul 30, 2018.

doi: 10.21037/acs.2018.08.03

View this article at: http://dx.doi.org/10.21037/acs.2018.08.03

\section{Introduction}

Functional impairment is a well-established consequence of critical illness, particularly among those with prolonged intensive care unit (ICU) stays in the context of sepsis, multi-organ failure, or prolonged mechanical ventilation $(1,2)$ and may persist well beyond the incident hospitalization. Perhaps the best-characterized example of this is with respiratory failure in the context of the acute respiratory distress syndrome (ARDS), where physical debility from neuromuscular weakness has been shown to persist for years beyond the initial insult $(3,4)$. Among the multifactorial etiologies of neuromuscular dysfunction, bed rest has been implicated as an important and potentially modifiable contributor (5). Further compounding the development of neuromuscular dysfunction in the ICU is the frequent use of sedation, particularly among patients requiring invasive mechanical ventilation for cardiopulmonary failure, often for the purpose of achieving adequate patient comfort, ventilator synchrony, cardiopulmonary stability and patient safety $(1,6)$.

In this context and in an effort to reduce the morbidity associated with critical illness, there has been an increased focus on interventions that minimize immobility and sedation in the ICU (7-11). A systematic review with metaanalysis of studies involving mobilization interventions in the ICU demonstrated that these interventions are safe, with a very low rate of potential safety events $(2.6 \%)$ and an even lower rate of consequences from these events (0.6\%) (12). There is conflicting data as to whether early mobilization 
in the ICU has a favorable impact on patient outcomes. While two prospective studies demonstrated favorable results of early mobilization on ICU and hospital length of stay, rates of delirium, and ventilator-free days compared to usual care $(7,13)$, others, including a randomized controlled trial of more intensive physical therapy in acute respiratory failure patients, have failed to demonstrate any effect on these same outcomes (14). A subsequent meta-analysis by Tipping et al. showed that active rehabilitation in the ICU was associated with more days alive and out of the ICU at 6 months, without any significant impact on mortality (15). With early mobilization shown to be achievable in the general critical care population, albeit with varying effects, there has been an interest in extending this strategy to those patients with the most severe forms of cardiopulmonary failure who are likely to be at greatest risk for the development of sequelae of critical illness. Generally, this population would be considered too unstable for mobilization because of extremes in cardiac and respiratory insufficiency. However, with the potential for extracorporeal life support (ECLS) to provide sufficient hemodynamic and gas exchange support (16), there is now greater opportunity to engage these patients in active physical therapy and early mobilization. Success with this approach is likely to be greatest when combined with efforts to minimize sedation and endotracheal intubation. The objectives of this review are to understand the current state of early mobilization for patients with cardiac failure supported with ECLS, the impact that ECLS configuration and patient management strategies may have on implementation and success of early mobilization and which patient populations might be best suited for and benefit most from early mobilization.

\section{Extracorporeal configurations and impact on mobilization}

ECLS consists of an extracorporeal circuit that has the ability to provide both gas exchange and circulatory support for patients with severe forms of cardiac or respiratory failure (16). The device consists of a gas exchange membrane through which venous blood is passed via a centrifugal pump. Within this gas exchanger, oxygen diffuses across a semipermeable membrane from a gas supply, known as sweep gas, into the blood, while carbon dioxide diffuses out of the blood. The well-oxygenated blood is then returned to the patient. Whether the device is providing gas exchange alone. or both gas exchange and hemodynamic support, is determined by the configuration of the device and where the blood is reinfused. For respiratory support, venovenous ECLS is the approach traditionally used, whereby deoxygenated venous blood is drained via a cannula placed in a central vein and oxygenated blood is reinfused back into a central vein (17). When ECLS is needed for hemodynamic support, such as in cases of severe, refractory cardiogenic shock, drainage still occurs via a central vein, but oxygenated blood is instead reinfused into the arterial system, referred to as venoarterial ECLS, thereby supplying blood flow directly into the systemic circulation (18).

The location of the ECLS cannulae will have a significant impact not only on the effectiveness of the circuit in providing cardiopulmonary support, but also on the ability to perform early mobilization. Venoarterial ECLS most commonly involves femoral cannulation for both drainage via a femoral vein and reinfusion via a femoral artery. Although early mobilization has been demonstrated to be safe in patients with other types of femoral catheters without any reported catheter-related complications $(19,20)$, ECLS cannulae are larger and perceived to be at a greater risk of complications, particularly arterial cannulae, than the types of catheters studied, making the avoidance of femoral ECLS cannulation preferable when mobilization is anticipated (21). Novel configuration strategies that avoid femoral cannulation in venoarterial extracorporeal membrane oxygenation (ECMO) include venous drainage via the internal jugular vein and reinfusion into the subclavian or innominate artery $(22,23)$ (Figure 1), with or without left ventricular apical cannulation for ventricular unloading purposes $(24,25)$. When left ventricular support is still required but gas exchange and right ventricular support are no longer needed, this hybrid configuration may be converted to an apical-axillary extracorporeal left ventricular assist device (VAD) by removing the venous drainage cannula and gas exchange membrane (24). The avoidance of sternotomy and need for cardiopulmonary bypass that would be employed with conventional surgical VADs makes this minimally invasive approach a more attractive option in its risk profile and further facilitates mobilization (25). Alternatively, a central ECLS approach (e.g., right atrial drainage and aortic reinfusion) may be considered when peripherally cannulated ECLS cannot provide sufficient support, though this approach necessitates a sternotomy (26). In patients requiring ECLS support in the setting of severe pulmonary hypertension with a concomitant inter-atrial communication (e.g., atrial septal defect), a scenario that would traditionally require venoarterial ECLS, a bicaval, dual-lumen cannula may be inserted through the internal 


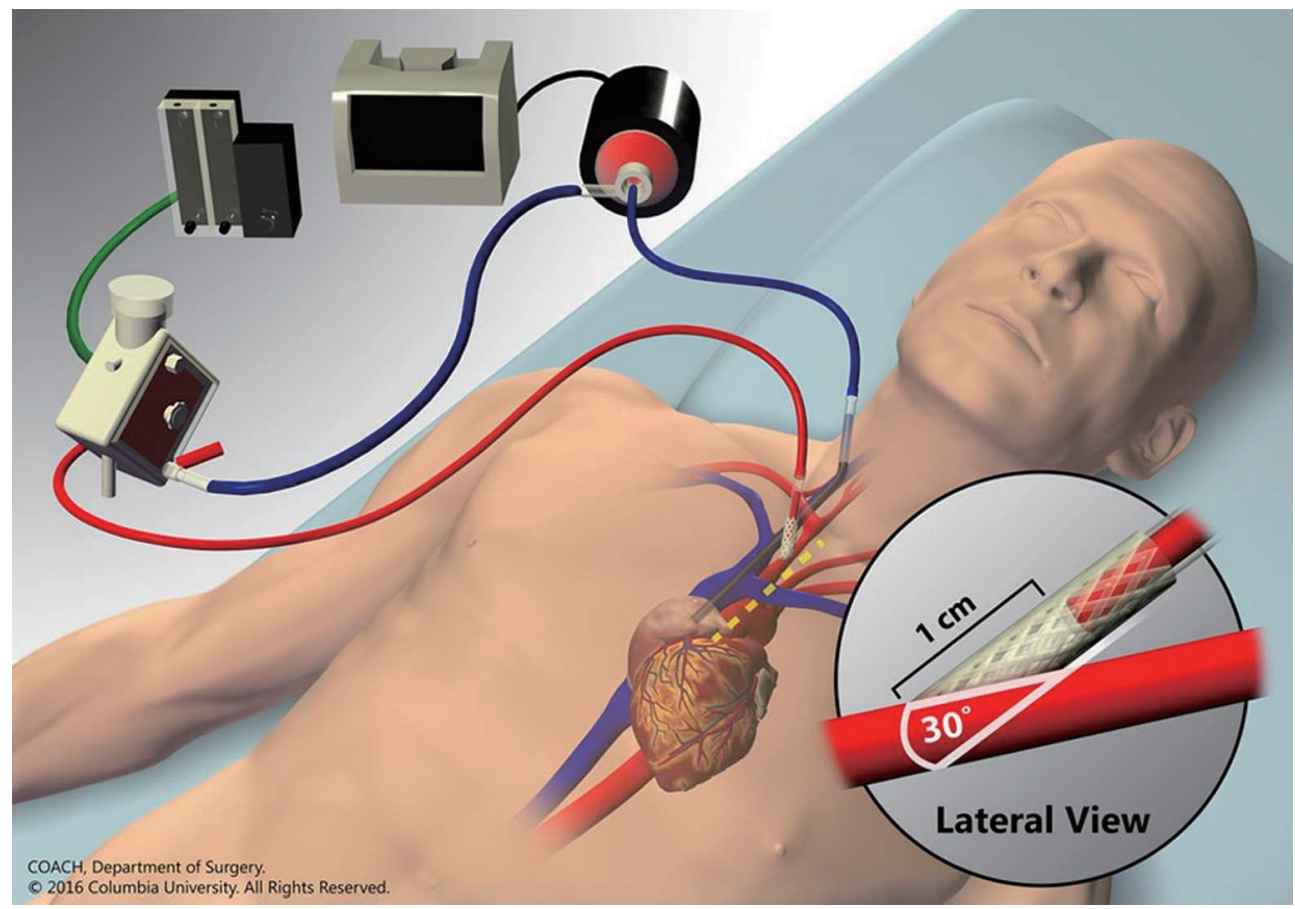

Figure 1 Venoarterial ECMO via internal jugular venous drainage and innominate arterial reinfusion through an end-to-side graft. Inset shows anastomosis angle from lateral view and dashed yellow line denotes location and extent of mini-sternotomy. Reproduced from Chicotka et al. (23) with permission from COACH Surgery at Columbia University. ECMO, extracorporeal membrane oxygenation.

jugular vein with the reinfusion jet directed across the defect, effectively creating an oxygenated right-to-left shunt and allowing for decompression of the right heart (27-29). The creation of an atrial septostomy in select patients with pulmonary hypertension may allow for the use of this approach as well $(30,31)$. An upper-body cannulation strategy is not limited to venoarterial ECLS. While a traditional venovenous ECLS approach is performed via two-site cannulation (most commonly femoral venous drainage and internal jugular venous reinfusion) (17), advances in cannula design have allowed for single-site cannulation with a bicaval, dual-lumen cannula for drainage both from the superior and inferior vena cava and reinfusion directed across the tricuspid valve (32), thereby avoiding the need for femoral cannulation.

In addition to novel cannulation strategies, there have been significant advances in the development of circuit components that are increasingly compact, including integrated systems in which the pump, membrane and console are contained within a single unit (33). While this compactness and portability of modern ECLS circuits have had perhaps their greatest impact on ease of inter-hospital transport for mobile ECMO transport teams $(34,35)$, they have simultaneously made it easier to achieve intra-hospital transport, including early mobilization (Figure 2) $(16,36)$.

\section{Patient selection and optimization for mobilization}

Extracorporeal support may be considered in cases of severe cardiac or respiratory failure. The potential for and prioritization of early mobilization will vary based on the underlying etiology and the intention of extracorporeal support, with use of an upper-body cannulation approach often preferred when mobility is of sufficiently high priority.

The feasibility and impact of early mobilization in the ECLS-supported cardiogenic shock population is poorly characterized in the literature, mostly due to the fact that the prognosis is often uncertain, with patients frequently receiving ECLS as a bridge to decision between recovery, VAD and transplantation. Although the existing data on mobilization in cardiac patients during ECLS is limited to small case series and anecdotal descriptions suggesting feasibility $(24-26,37)$, there is an expanding repository of 


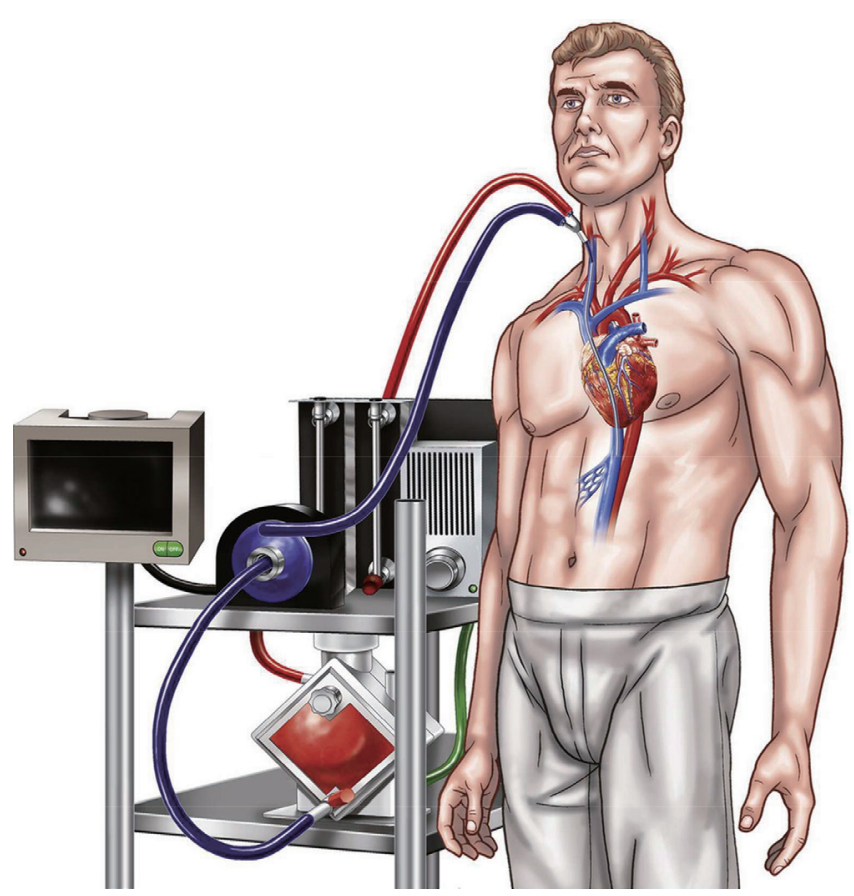

Figure 2 An awake, extubated strategy, combined with an upperbody configuration and compact circuitry, facilitates mobilization in patients with cardiopulmonary failure requiring extracorporeal membrane oxygenation (ECMO). Reproduced with permission from Abrams et al. (16).

published literature in the respiratory ECLS population, from which data may be extrapolated to potentially justify a comparable approach in cardiac patients.

Owing to the need for the maintenance of physical fitness to ensure there is sufficient physiological reserve to survive and recover from transplant surgery, the greatest focus of early mobilization during ECLS has been in the bridge to transplant (BTT) population. In fact, good rehabilitation potential has been cited among the characteristics for which pre-lung transplant ECLS has been recommended in the International Society for Heart and Lung Transplantation guidelines (38). Not only should patients already receiving ECLS as BTT be prioritized for mobilization, but as physical deconditioning and immobilization are considered strong relative contraindications to transplant candidacy, ECLS should be considered as a means of optimizing physical rehabilitation before significant debility develops as a result of severe cardiopulmonary failure and impaired exercise capacity (39). Although the pre-lung transplant ECLS mobilization data mostly focus on patients with isolated respiratory failure, there is emerging data for the subset of patients with pulmonary hypertension and concomitant right ventricular failure, from which parallels may be drawn to the more commonly encountered leftsided cardiogenic shock population $(28,29,40,41)$.

An early mobilization strategy has been shown in the literature to confer an advantage for successful bridge to lung transplant (42-44). In a cohort of 72 patients receiving ECLS as bridge to transplantation, in which an awake, active physical therapy approach was prioritized, $69.4 \%$ of patients ambulated while receiving ECLS support (42). The rate of ambulation, perhaps not surprisingly, was significantly higher among patients who survived to transplantation than those who did not survive $(80 \% v s$. $56.2 \%, \mathrm{P}=0.03)$. Among the patients who were successfully transplanted but did not ambulate $(n=8)$, all patients were able to perform active physical therapy at the bedside and were on ECLS support for less than 7 days prior to transplantation. Patient selection and mobilization practices remain critical components to optimization of outcomes. Patients with higher severity of illness and who are maintained on sedation and immobilized during ECLS support pre-transplant may be at risk for greater neuromuscular dysfunction and longer length of stay posttransplant, if in fact they remain appropriate transplant candidates at all (45).

Patients awaiting heart or lung transplantation, particularly those with end organ failure severe enough to warrant consideration of ECLS, are often supported with or considered for invasive mechanical ventilation. In the cardiac failure population, the need for invasive mechanical ventilation will depend on the degree of gas exchange impairment, often due to cardiogenic pulmonary edema or concomitant non-cardiogenic respiratory failure (e.g., ARDS, pneumonia, etc.). The pre-transplant use of invasive mechanical ventilation, irrespective of etiology and independent of the use of ECLS, has traditionally been associated with worse post-transplant outcomes than patients not requiring invasive mechanical ventilation, attributed at least in part to higher pre-transplant severity of illness and deconditioning associated with immobility (46-49). However, the use of invasive mechanical ventilation also inherently puts patients at risk for ventilator-associated complications, including ventilator-associated pneumonia, ventilator-associated lung injury, dynamic hyperinflationwith the potential to cause further hemodynamic compromise-and suboptimal delivery of nutrition and inhaled medications $(50,51)$. In select patients who might otherwise require invasive mechanical ventilation, ECLS 
may provide sufficient gas exchange support to allow for the removal, or avoidance altogether, of the ventilator, which eliminates ventilator-associated complications, facilitates removal of sedation, and may further increase the likelihood of mobilization $(36,42,43,52,53)$. In patients who, despite ECLS, do not tolerate extubation and have an ongoing need for invasive mechanical ventilation, early tracheostomy placement may be considered to maximize patient comfort and, when necessary, facilitate airway clearance. The need for tracheostomy and mechanical ventilation should not preclude efforts to perform early mobilization (42). In fact, prior cohort studies have demonstrated successful performance of active physical therapy by pre-transplant patients despite $47 \%$ undergoing tracheostomy placement and $32 \%$ remaining on invasive mechanical ventilation during ECLS support (36).

Pulmonary hypertension with right ventricular failure represents a distinct cardiac failure population in whom ECLS is emerging as a strategy of BTT and occasionally, as bridge to recovery (BTR) when there is an opportunity to treat reversible causes of decompensation and optimize pulmonary hypertension-targeted therapies $(28,29,41)$. An awake and mobile ECLS approach has been shown to be feasible in this population, though the effect of this combined strategy on outcomes, other than successful bridging to transplantation in small case series, has yet to be evaluated. Because this patient population is at risk for rapid decompensation in the setting of abrupt changes in pulmonary vascular resistance, intra-thoracic pressure and right ventricular volume status, careful consideration must be made with regards to endotracheal intubation when there is an apparent need for mechanical ventilatory support. One consideration for these patients is placement of femoral venoarterial ECLS in lieu of intubation, providing both right ventricular unloading and gas exchange support. However, this approach may be insufficient at delivering oxygenated blood to the upper body, particularly because these patients typically have preserved left ventricular function and impaired native gas exchange (54). Reinfused oxygenated blood from the ECLS circuit is impeded by native cardiac output, resulting in predominantly deoxygenated blood being supplied to the ascending aorta and the coronary and carotid arteries. Furthermore, the femoral cannulae may limit mobilization. An upper-body venoarterial ECLS configuration mostly alleviates these issues and in carefully selected patients, such an approach has been demonstrated to be feasible without the need for peri-operative intubation (55).
Early mobilization in cardiopulmonary failure is not limited to the BTT population, although the benefit of such a strategy is less well defined in other cohorts $(21,36,56)$. Patients receiving ECLS as a BTR may be candidates for an awake, mobile strategy, although they are inherently more severely critically ill than patients with acute cardiopulmonary failure managed without ECLS support who have been more traditional candidates for mobilization $(13,36,56)$. Data for mobilization of patients with severe cardiac failure supported with ECLS as BTR is scarce. While the hemodynamic status and consequences of mobilization may differ, some useful information may be gleaned from the existing data on early mobilization of patients receiving ECLS as BTR from severe respiratory failure, particularly given the comparable demand on the respiratory system that occurs during mobilization. If early mobilization, including ambulation, can be achieved in a population with ECLS-supported severe, but potentially reversible, gas exchange impairment, there is reasonable promise for mobilization to be achievable in those with potentially reversible cardiac failure supported with ECLS. Whether the application of mobilization has a favorable impact on outcomes has yet to be elucidated.

Patients with severe respiratory failure requiring invasive mechanical ventilation are often managed with deep sedation and neuromuscular blockade to maintain adequate gas exchange. The support provided by ECLS may be sufficient to allow for removal of these medications, thereby promoting wakefulness and facilitating the potential removal of invasive mechanical ventilation. In a cohort of 61 patients with ARDS supported with ECLS, not only was consultation with an ICU physiotherapist associated with a significantly lower odds of death (OR 0.19, 95\% CI: 0.04$0.98, \mathrm{P}=0.048)$, but a sedation-agitation score consistent with being calm and cooperative correlated with higher intensity physiotherapy, which both reinforces the favorable impact of an awake ECLS strategy on participation in mobilization and highlights that such an approach may only be achievable in select patients.

Regarding the potential impact of early mobilization on discharge disposition, two single-center cohorts of patients with ECLS-supported cardiopulmonary failure who engaged in active physical therapy demonstrated high rates of discharge to home or acute rehabilitation $(21,36)$. The lack of a comparison group in either study calls into question the impact of early mobilization itself on these outcomes.

Although there is an emphasis on upper-body cannulation 
Initial assessments

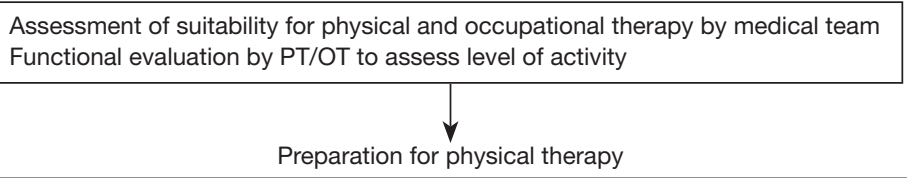

Secure all intravenous lines, remove extraneous

Ensure adequate portable oxygen supply (respiratory therapist as needed)

Ensure hemodynamic monitoring available throughout treatment Inspect extracorporeal circuit

- Ensure adequate tubing slack to allow safe movement of patient without undue strain on circuit

- Tighten all connections

- Assess for leaks and thromboses within tubing

Assemble and brief the team (PT, OT, nurse, perfusionist, nurse practitioner)

Initiation of physical therapy

Responsibilities:

- Monitoring vital signs: PT, OT, and nurse

- Monitoring circuit integrity: perfusionist

- Assisting the patient and providing contact guard: PT, OT

Figure 3 Multidisciplinary, stepwise approach to physical therapy in the medical intensive care unit. Reproduced with permission from Abrams et al. (36).

to facilitate mobilization, this need not be the exclusive approach when considering early mobilization, which may have important implications for cardiac failure patients in whom femoral venoarterial ECLS is performed under emergent conditions. A cohort study of ECLS-supported patients with respiratory failure $(n=35)$ demonstrated successful ambulation of 2 patients with femoral cannulae (36) and a larger single-center cohort of patients receiving ECLS as BTR ( $\mathrm{n}=167)$ demonstrated successful mobilization out of bed in 13 patients with bifemoral cannulae without cannulaassociated complications (21), highlighting the potential safety of mobilizing patients out of bed with either venous or arterial femoral cannulation. However, the relatively frequent concomitant use of femoral access for an intraaortic balloon pump or a percutaneous VAD for ventricular unloading in cardiac failure patients adds additional complexity.

Despite the apparent safety, feasibility and success of active physical therapy in patients receiving ECLS as BTR at highly specialized, high volume ECLS centers, there are no studies prospectively assessing the effect of active physical therapy and early mobilization on this patient population compared to usual care. A prospective pilot study of ECLS patients randomized to early physical therapy or standard care aims to evaluate the impact of physical therapy on functional recovery (57).

Consideration of an awake, mobile strategy may potentially be extended to other forms of mechanical circulatory support beyond ECLS. Those supported with short-term surgical VADs should be prioritized for mobilization whenever feasible, as has been described in cohorts of patients supported with apical-axillary VADs and temporary, conventional surgical biventricular VADs $(25,37)$. Percutaneous coaxial VADs may be less amenable to mobilization due to the relatively greater risk of device displacement during physical activity, though mobilization when such devices are placed through the axilla may be more feasible (58).

A universal theme throughout the existing literature of early mobilization for both cardiac and respiratory failure populations is the implementation of a multidisciplinary team approach to ensure safe and successful performance of mobility with patients receiving ECLS (Figure 3) $(21,36,37,56)$. A coordinated effort by intensivists, surgeons, nurses, ECLS specialists, physical and occupational therapists and respiratory therapists-when appropriatehave been reported and are encouraged $(21,36,56)$. The impact of a multidisciplinary early mobilization strategy on safety is highlighted by the low rate of complications in the largest reported cohort study to date-607 physical therapy session, $<0.5 \%$ event rate, all minor (21). To further ensure optimal safety and outcomes, guidelines or protocols outlining the process of early mobilization should be created and followed, with well-delineated roles and responsibilities 
for each participant and discipline (36). Particular attention must be paid to cannula positioning and patient stability. In ECLS for cardiac failure, in which both venous and arterial cannulae are utilized, adequate securement of cannulae prior to mobilization is of the utmost importance. Cannulae for venous drainage are typically longer, whereas arterial reinfusion cannulae usually have a shorter length and may be at greater risk for dislodgement. It is crucial to be vigilant of any interruption of blood flow due to kinks or bends in cannulae, particularly in the context of activities involving hip flexion. The wire reinforcement component of cannulae may help guard against such occurrences.

Whereas gas exchange is often the parameter of greatest concern for respiratory failure patients being mobilized, hemodynamic instability is more likely to develop during mobilization of those with cardiac failure. Although not well described in the literature, patients with cardiac failure may require an increase in ECLS support, particularly blood flow, during active physical therapy to match their physiological needs, depending on the strenuousness of the therapy session and the patient's physiological reserve. Additionally, there may be a role for the initiation or escalation of inotropic or vasopressor support if ECLS support alone is insufficient during physical therapy. However, if a patient requires escalation of inotropic or vasopressor support solely to achieve mobilization, then one must consider whether the theoretical benefit of mobilization outweighs the potential risks. If early mobilization is anticipated during ECLS support, then consideration should be made in choosing cannulae at the time of ECLS initiation that can achieve adequate blood flow to match the patient's expected physiological needs.

\section{Limitations and future directions}

When considering the role of early mobilization in patients with cardiac failure supported with ECLS, it is important to recognize the limitations in the existing data, which by and large consist of small case series or cohort studies without comparator groups, owing to the limited experience with such an approach and the complexity of these patients. Future prospective randomized trials may help clarify the role of early mobilization in patients receiving ECLS (57). However, the specialized nature of this intervention and the greater potential risk profile in the cardiac failure population, compared to those with respiratory failure, may ultimately limit the broader applicability of such a strategy for those receiving ECLS for cardiac indications.

\section{Conclusions}

An awake and mobile ECLS strategy is safe and feasible in patients receiving extracorporeal support for advanced cardiac and respiratory failure. For patients awaiting transplantation, in whom adequate physical conditioning is a prerequisite for transplant candidacy, early mobilization should be a high priority. Future studies will help clarify the impact of early mobilization in the ECLS BTR population and whether the benefit seen in the non-ECLS-supported critically ill population can be expected in ECLS patients. Irrespective of the etiology and expected outcome, early mobilization should be performed by a multidisciplinary team with adequate experience and well-defined roles and responsibilities. When feasible, endotracheal extubation may further facilitate successful mobilization.

\section{Acknowledgements}

Funding: Dr. Garan is supported by National Institutes of Health Grant No. KL2TR001874, and has previously received honoraria from Abiomed (Danvers, MA).

\section{Footnote}

Conflicts of Interest: Dr. Brodie is currently the co-chair of the Trial Steering Committee for the VENT-AVOID trial sponsored by ALung Technologies. He was previously on the medical advisory board of ALung Technologies and Kadence (Johnson \& Johnson). All compensation for these activities is paid to Columbia University. Dr. Garan is now an unpaid consultant for Abiomed. Dr. Abrams has no conflicts of interest to declare.

\section{References}

1. Needham DM. Mobilizing patients in the intensive care unit: improving neuromuscular weakness and physical function. JAMA 2008;300:1685-90.

2. Stevens RD, Dowdy DW, Michaels RK, et al. Neuromuscular dysfunction acquired in critical illness: a systematic review. Intensive Care Med 2007;33:1876-91.

3. Herridge MS, Tansey CM, Matte A, et al. Functional disability 5 years after acute respiratory distress syndrome. N Engl J Med 2011;364:1293-304.

4. Dowdy DW, Eid MP, Dennison CR, et al. Quality of life 
after acute respiratory distress syndrome: a meta-analysis. Intensive Care Med 2006;32:1115-24.

5. Berg HE, Larsson L, Tesch PA. Lower limb skeletal muscle function after $6 \mathrm{wk}$ of bed rest. J Appl Physiol 1997;82:182-8.

6. Weinert CR, Calvin AD. Epidemiology of sedation and sedation adequacy for mechanically ventilated patients in a medical and surgical intensive care unit. Crit Care Med 2007;35:393-401.

7. Morris PE, Goad A, Thompson C, et al. Early intensive care unit mobility therapy in the treatment of acute respiratory failure. Crit Care Med 2008;36:2238-43.

8. Kress JP, Pohlman AS, O'Connor MF, et al. Daily interruption of sedative infusions in critically ill patients undergoing mechanical ventilation. $\mathrm{N}$ Engl J Med 2000;342:1471-7.

9. Kress JP, Gehlbach B, Lacy M, et al. The long-term psychological effects of daily sedative interruption on critically ill patients. Am J Respir Crit Care Med 2003;168:1457-61.

10. De Jonghe B, Bastuji-Garin S, Fangio P, et al. Sedation algorithm in critically ill patients without acute brain injury. Crit Care Med 2005;33:120-7.

11. Wahab R, Yip NH, Chandra S, et al. The implementation of an early rehabilitation program is associated with reduced length of stay: A multi-ICU study. J Intensive Care Soc 2016;17:2-11.

12. Nydahl P, Sricharoenchai T, Chandra S, et al. Safety of Patient Mobilization and Rehabilitation in the Intensive Care Unit. Systematic Review with Meta-Analysis. Ann Am Thorac Soc 2017;14:766-77.

13. Schweickert WD, Pohlman MC, Pohlman AS, et al. Early physical and occupational therapy in mechanically ventilated, critically ill patients: a randomised controlled trial. Lancet 2009;373:1874-82.

14. Morris PE, Berry MJ, Files DC, et al. Standardized Rehabilitation and Hospital Length of Stay Among Patients With Acute Respiratory Failure: A Randomized Clinical Trial. JAMA 2016;315:2694-702.

15. Tipping CJ, Harrold M, Holland A, et al. The effects of active mobilisation and rehabilitation in ICU on mortality and function: a systematic review. Intensive Care Med 2017;43:171-83.

16. Abrams D, Combes A, Brodie D. Extracorporeal Membrane Oxygenation in Cardiopulmonary Disease in Adults. J Am Coll Cardiol 2014;63:2769-78.

17. Brodie D, Bacchetta M. Extracorporeal membrane oxygenation for ARDS in adults. $\mathrm{N}$ Engl J Med
2011;365:1905-14.

18. Abrams D, Garan AR, Abdelbary A, et al. Position paper for the organization of ECMO programs for cardiac failure in adults. Intensive Care Med 2018;44:717-29.

19. Damluji A, Zanni JM, Mantheiy E, et al. Safety and feasibility of femoral catheters during physical rehabilitation in the intensive care unit. J Crit Care 2013;28:535.e9-15.

20. Perme C, Nalty T, Winkelman C, et al. Safety and Efficacy of Mobility Interventions in Patients with Femoral Catheters in the ICU: A Prospective Observational Study. Cardiopulm Phys Ther J 2013;24:12-7.

21. Wells CL, Forrester J, Vogel J, et al. Safety and Feasibility of Early Physical Therapy for Patients on Extracorporeal Membrane Oxygenator: University of Maryland Medical Center Experience. Crit Care Med 2018;46:53-9.

22. Biscotti M, Bacchetta $M$. The "sport model": extracorporeal membrane oxygenation using the subclavian artery. Ann Thorac Surg 2014;98:1487-9.

23. Chicotka S, Rosenzweig EB, Brodie D, et al. The "Central Sport Model": Extracorporeal Membrane Oxygenation Using the Innominate Artery for Smaller Patients as Bridge to Lung Transplantation. ASAIO J 2017;63:e39-44.

24. Takeda K, Garan AR, Topkara VK, et al. Novel minimally invasive surgical approach using an external ventricular assist device and extracorporeal membrane oxygenation in refractory cardiogenic shock. Eur J Cardiothorac Surg 2017;51:591-6.

25. Takeda K, Garan AR, Ando M, et al. Minimally invasive CentriMag ventricular assist device support integrated with extracorporeal membrane oxygenation in cardiogenic shock patients: a comparison with conventional CentriMag biventricular support configuration. Eur J Cardiothorac Surg 2017;52:1055-61.

26. Shudo Y, Wang H, Ha RV, et al. Heart transplant after profoundly extended ambulatory central venoarterial extracorporeal membrane oxygenation. J Thorac Cardiovasc Surg 2018;156:e7-9.

27. Javidfar J, Brodie D, Sonett J, et al. Venovenous extracorporeal membrane oxygenation using a single cannula in patients with pulmonary hypertension and atrial septal defects. J Thorac Cardiovasc Surg 2012;143:982-4.

28. Abrams DC, Brodie D, Rosenzweig EB, et al. Upperbody extracorporeal membrane oxygenation as a strategy in decompensated pulmonary arterial hypertension. Pulm Circ 2013;3:432-5.

29. Rosenzweig EB, Brodie D, Abrams DC, et al. Extracorporeal membrane oxygenation as a novel bridging 
strategy for acute right heart failure in group 1 pulmonary arterial hypertension. ASAIO J 2014;60:129-33.

30. Camboni D, Akay B, Sassalos P, et al. Use of venovenous extracorporeal membrane oxygenation and an atrial septostomy for pulmonary and right ventricular failure. Ann Thorac Surg 2011;91:144-9.

31. Hoopes CW, Gurley JC, Zwischenberger JB, et al. Mechanical Support for Pulmonary Veno-occlusive Disease: Combined Atrial Septostomy and Venovenous Extracorporeal Membrane Oxygenation. Semin Thorac Cardiovasc Surg 2012;24:232-4.

32. Javidfar J, Brodie D, Wang D, et al. Use of bicaval duallumen catheter for adult venovenous extracorporeal membrane oxygenation. Ann Thorac Surg 2011;91:17638; discussion 1769.

33. Lehle K, Philipp A, Hiller KA, et al. Efficiency of gas transfer in venovenous extracorporeal membrane oxygenation: analysis of 317 cases with four different ECMO systems. Intensive Care Med 2014;40:1870-7.

34. Stephens RS, Abrams D, Brodie D. Inter-hospital transport on extracorporeal membrane oxygenation. In: Vincent JL. editor. Annual Update in Intensive Care and Emergency Medicine. Netherlands: Springer International Publishing, 2018.

35. Biscotti M, Agerstrand C, Abrams D, et al. One Hundred Transports on Extracorporeal Support to an Extracorporeal Membrane Oxygenation Center. Ann Thorac Surg 2015;100:34-9; discussion 39-40.

36. Abrams D, Javidfar J, Farrand E, et al. Early mobilization of patients receiving extracorporeal membrane oxygenation: a retrospective cohort study. Crit Care 2014;18:R38.

37. McGarrigle L, Caunt J. Physical Therapist-Led Ambulatory Rehabilitation for Patients Receiving CentriMag Short-Term Ventricular Assist Device Support: Retrospective Case Series. Phys Ther 2016;96:1865-73.

38. Weill D, Benden C, Corris PA, et al. A consensus document for the selection of lung transplant candidates: 2014--an update from the Pulmonary Transplantation Council of the International Society for Heart and Lung Transplantation. J Heart Lung Transplant 2015;34:1-15.

39. Abrams D, Brodie D, Arcasoy SM. Extracorporeal Life Support in Lung Transplantation. Clin Chest Med 2017;38:655-66.

40. Chicotka S, Pedroso FE, Agerstrand CL, et al. Increasing Opportunity for Lung Transplant in Interstitial Lung Disease with Pulmonary Hypertension. Ann Thorac Surg 2018;106:1812-9.
41. Shudo Y, Kasinpila P, Lee AM, et al. Ambulating femoral venoarterial extracorporeal membrane oxygenation bridge to heart-lung transplant. J Thorac Cardiovasc Surg 2018;156:e135-7.

42. Biscotti M, Gannon WD, Agerstrand C, et al. Awake Extracorporeal Membrane Oxygenation as Bridge to Lung Transplantation: A 9-Year Experience. Ann Thorac Surg 2017;104:412-9.

43. Fuehner T, Kuehn C, Hadem J, et al. Extracorporeal membrane oxygenation in awake patients as bridge to lung transplantation. Am J Respir Crit Care Med 2012;185:763-8.

44. Hoopes CW, Kukreja J, Golden J, et al. Extracorporeal membrane oxygenation as a bridge to pulmonary transplantation. J Thorac Cardiovasc Surg 2013;145:8627; discussion 867-8.

45. Hayes K, Hodgson CL, Pellegrino VA, et al. Physical Function in Subjects Requiring Extracorporeal Membrane Oxygenation Before or After Lung Transplantation. Respir Care 2018;63:194-202.

46. Christie JD, Edwards LB, Kucheryavaya AY, et al. The Registry of the International Society for Heart and Lung Transplantation: 29th adult lung and heart-lung transplant report-2012. J Heart Lung Transplant 2012;31:1073-86.

47. Mason DP, Thuita L, Nowicki ER, et al. Should lung transplantation be performed for patients on mechanical respiratory support? The US experience. J Thorac Cardiovasc Surg 2010;139:765-73.e1.

48. Toyoda Y, Bhama JK, Shigemura N, et al. Efficacy of extracorporeal membrane oxygenation as a bridge to lung transplantation. J Thorac Cardiovasc Surg 2013;145:1065-71.

49. George TJ, Beaty CA, Kilic A, et al. Outcomes and temporal trends among high-risk patients after lung transplantation in the United States. J Heart Lung Transplant 2012;31:1182-91.

50. Heyland DK, Cook DJ, Griffith L, et al. The attributable morbidity and mortality of ventilator-associated pneumonia in the critically ill patient. The Canadian Critical Trials Group. Am J Respir Crit Care Med 1999;159:1249-56.

51. Abrams DC, Brenner K, Burkart KM, et al. Pilot study of extracorporeal carbon dioxide removal to facilitate extubation and ambulation in exacerbations of chronic obstructive pulmonary disease. Ann Am Thorac Soc 2013;10:307-14.

52. Olsson KM, Simon A, Strueber M, et al. Extracorporeal membrane oxygenation in nonintubated patients as bridge to lung transplantation. Am J Transplant 2010;10:2173-8. 
53. Abrams D, Brodie D. Emerging indications for extracorporeal membrane oxygenation in adults with respiratory failure. Ann Am Thorac Soc 2013;10:371-7.

54. Biscotti M, Lee A, Basner RC, et al. Hybrid Configurations via Percutaneous Access for Extracorporeal Membrane Oxygenation: A Single Center Experience. ASAIO J 2014;60:635-42.

55. Biscotti M, Vail E, Cook KE, et al. Extracorporeal Membrane Oxygenation with Subclavian Artery Cannulation in Awake Patients with Pulmonary Hypertension. ASAIO J 2014;60:748-50.

56. Munshi L, Kobayashi T, DeBacker J, et al. Intensive Care Physiotherapy during Extracorporeal Membrane

Cite this article as: Abrams D, Garan AR, Brodie D. Awake and fully mobile patients on cardiac extracorporeal life support. Ann Cardiothorac Surg 2019;8(1):44-53. doi: 10.21037/ acs.2018.08.03
Oxygenation for Acute Respiratory Distress Syndrome. Ann Am Thorac Soc 2017;14:246-53.

57. Australian and New Zealand Intensive Care Research Centre. Extracorporeal Membrane Oxygenation Physical Training (ECMO-PT). In: ClinicalTrials.gov. Bethesda (MD): National Library of Medicine (US). 2000. [cited 2018 Apr 10]. Available online: http://clinicaltrials.gov/ ct2/show/NCT03328767

58. Esposito ML, Jablonski J, Kras A, et al. Maximum level of mobility with axillary deployment of the Impella 5.0 is associated with improved survival. Int J Artif Organs 2018;41:236-9. 